\title{
Robotic Monocular SLAM in Wastewater Treatment Plants with a Sampling Device
}

\author{
Edmundo Guerra $^{1}$, Yolanda Bolea ${ }^{1}$, Rodrigo Munguia ${ }^{2}$, Antoni Grau ${ }^{1}$ \\ ${ }^{1}$ Automatic Control Dept, BarcelonaTech, 08034 Barcelona, Spain \\ 2 Dept of Computer Science, CUCEI, Univ. of Guadalajara, Guadalajara 44430, Mexico \\ \{edmundo.guerra, yolanda.bolea, rodrigo.munguia, antoni.grau\}@upc.edu
}

\begin{abstract}
A novel application is presented in this paper. Wastewater treatment plants need to sample water to follow the cleaned process continuously. This task is very tedious for laboratory workers and, mainly, can be done in reduced areas of the large basins that form the whole plant, specifically only in the edges of those basins. The new proposal is to enlarge the sampling area and reduce the load for workers. A new unmanned aerial vehicle has been designed together with a novel tool to take water samples. Moreover, a new architecture for the mission plan is presented in form of multi-agents. Experiments have been done in order to test the feasibility of the proposal.
\end{abstract}

Keywords: monocular SLAM HRI mobile robotics wastewater treatment plant

\section{Introduction}

Management and supervision techniques for industrial scale processes have historically been driven by the changes produced by technical innovations. With each increase in efficiency and scale due improvements in the technologies used to produce the good and services, an increased production had to be managed. In these production tasks the automation of processes has made the human work (as physical effort) largely redundant in the heavier tasks. For more complex tasks human presence is still required, given the enormous versatility of the human cognitive abilities when compared with automated systems, which must be built according to precise specifications, and require great efforts to implement any change. This kind of tasks is especially prevalent in supervision, monitoring and management functions, where the expertise provided by human elements in a system is extremely difficult to model in a general way. In this context, the best results achieved are specialized support tools 1 , which routinely can beat human in specific well-delimited tasks, but lack generality and flexibility to replace a human.

These monitoring and supervision tasks can present repetitive operations which could be subject to automation thanks to the increased pace of autonomous robotics development observed in the last decades. The last revolutions in the field of autonomous robotics is coming in the form of unmanned aerial vehicles (UAV) which, thanks to developments in battery technology, microelectromechanical systems (MEMS), and 
sensor technologies including IMU (inertial measurement unit), GPS, RFs, have gained a presence in several industries, like specialized logistic services, audio-visual production, construction surveying, etc... Still, in this industries the UAVs operate always under human direct control, or at least supervision, in most cases, with limited autonomous applications, and only in the surveying field are gaining a significant foothold [1] [2] [3] [4]. Applications in this industry often benefit greatly from their mobility and freedom of movement, an in many cases, supposes a reduction in the risks assumed by human workers.

In order to automatize these operations, the robotic UAVs have to have autonomy of operation enough to act on their own agency: they will have to take decisions with respect a known task and environment that are still dynamic and cannot be completely modelled and represent. There are several design approaches [5] and technologies [6] when trying to produce robots with these capabilities, being one of the most fruitful the multi-agent system (MAS). A good example of how to exploit all these features in a robotics MAS can be found in [7], where based on MAS methodologies [8], a solution for a surveying surface vehicle is implemented over the ROS framework [9].

With respect to the problem of sampling and autonomous robots, in previous works [10] authors presented a general architecture for a single unmanned aerial vehicle working jointly with a unmanned surface vehicle in a wastewater plant. This work sets the basis of this new research, where a UAV is part of a sensor network with a newly designed sampling probe to take samples from any point in wastewater tanks and basins. The new system supports a network of UAV platforms, whose design architecture is presented as a contribution, like its role as a sensor in a network sensor, the new custom sampling probe, and several results obtained during the development and testing of a prototype UAV. The rest of this work is structured as follows: the first section describes with more detail the sampling problem, the reference use case scenario considered for this work, and describes from with a high level point of view how the system works. The following section, "System and Hardware Structure" describes which components build the systems, how they are organized in subsystems, and put emphasis in the built UAV prototype, detailing its hardware and the organization in terms of connection and interfaces. Next section, "System design and software architecture" deals with the design of the system from the MAS point of view, how the software is (from a logical view) organized and implemented, and provides justification when the logical designs does not fit the actual implementation. This is followed by the "Experimental results" section, where the results obtained during the development and testing phase are discussed, especially for the localization problem of the unmanned aerial vehicle; in this section the resulting sample collector probe is presented and discussed as well. To conclude, the work presented is discussed in terms of the contributions made, and the most promising lines of work are commented. 


\section{UAV Network Sampling of Wastewater Plant}

\subsection{Wastewater Plant Sampling}

In terms of management, one of the most critical processes to properly monitor a wastewater treatment plant is the evaluation of processed water, to ensure its compliance with the required specifications. The factors to be accounted and monitored include from chemical properties of the water, like $\mathrm{pH}$, to concentrations of biological and inorganic pollutants. This frequently means obtaining multiple samples, with variable specifications (e.g.: samples for microbiological testing cannot be exposed to sunlight, nitric nitrogen samples must be processed within 2 hours, etc...) [11] [12] [13]. Periodic testing requirements in this case means planning account for both the requisites and the resources available, be it technical or human.

For most of the metrics to test, both for clean waters and wastewaters, one of the most desired features is the homogeneity of the water to be sampled. This guarantees that the results obtained are representative of the water present in a given basin or tank, providing validity to the analyses. Notice that process tanks in water treatment plants present slow dynamics, with low flow velocities that drag dilution processes along all the tank. While these processes help homogenize the concentration of several pollutants and equalizes chemical indicators (increasing homogeneity of the sample and accuracy of the test), the low speed makes the tanks and basins to act as buffers. This fact produces delays hard to model, where water subjected to different rates of treatment and dilutive processes due entering the tanks at separated times can be present concurrently in the tank. In turn, this variability in the state of the water means that proper representative sampling of a single basin may require several samples at different points. This requirement may complicate the sampling process, as the optimal points for sampling from an analytic point of view may be hard to access, requiring special equipment or structures to reach them.

These features of the sampling process may present additional challenges for human workers beyond the accessibility of the areas, as in certain environments it is possible to find noxious gasses and substances, the samples may present special requirements (as commented above, with respect to timing, exposition, insulation...), and the weather or other factors can introduce further hindrances (excessive temperatures, insufficient lighting, dangerous conditions due wind speeds...). All these factors increase the complexity of the sampling procedures and the risks associated, especially for human operators. These complexities and risks may be managed with improvements in methodologies, resources and installations, but at the end they may only reduce risks, not remove them as long as the sample collection is performed by human workers.

\subsection{Network Sampling Environment}

For the proposed network sampling system, a medium sized wastewater processing plant is considered (see Fig 1). The plant of Sant Feliu (Barcelona) services 320,000 inhabitants and relevant infrastructure and economic activities. It can process up to $72,000 \mathrm{~m} 3$ of wastewater, coming mainly from 2 sources: domestic sewage, originated at residential housing and commercial areas, without special pollutants; and industrial 
wastewater, pre-treated by the industries to remove specific pollutants, and returned afterwards to the conventional wastewater circuit.

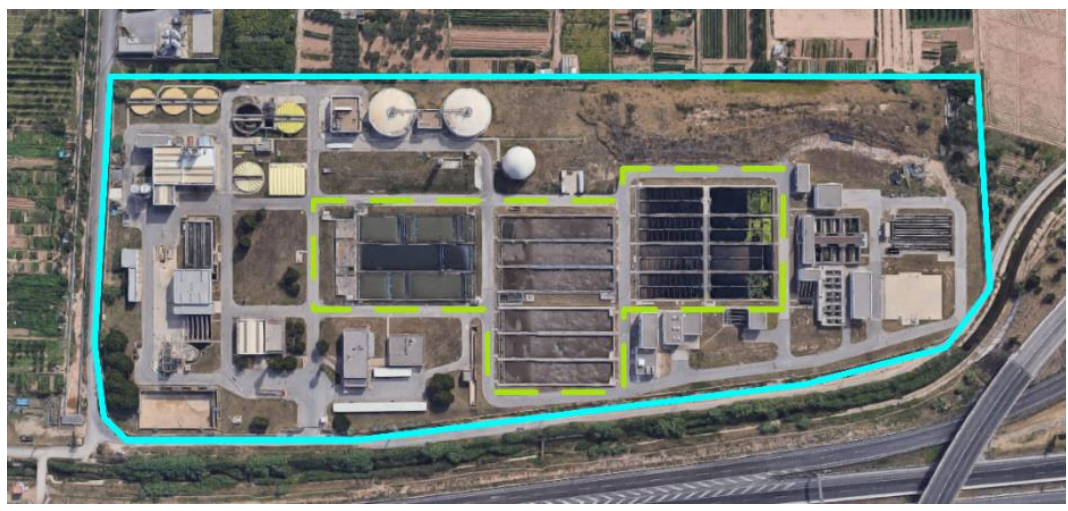

Fig. 1. Delayed Inverse-Depth (DI-D) Monocular EKF-SLAM Satellite view of the wastewater plant considered, located 10km south-west from Barcelona, in Sant Feliu municipality. The plant compound measures $480 \mathrm{~m}$ long by $192 \mathrm{~m}$ wide (enclosed in light blue line). The open air basins area, where the network sampling system is designed to operate, is enclosed by the dashed green line, making $250 \mathrm{~m}$ long by $118 \mathrm{~m}$ wide. The basins present several sizes, with lengths varying between $35 \mathrm{~m}$ and $65 \mathrm{~m}$, and widths in the range between $11.5 \mathrm{~m}$ and $13.5 \mathrm{~m}$.

Water processed by the plant is expected to reach the quality metrics required by regulatory bodies to allow its return to the environment. Several processes are applied successively, from mechanical filtering and decanting, to acceleration of organic matter degradation. As part of the resources required for these processes, 17 outdoor open air tanks and basins are present, with an accumulated surface over 11,200m2.

\subsection{UAV Network Sampling}

The proposed system offers a solution for the sampling and the analyses required to monitor and supervise the open air basins in a wastewater treatment plant. The sampling network allows to analyse the water present in almost every point of the open basins and tanks, thus, acting as a virtual network of multiparametric sensors with hundreds of probes deployed. To achieve this goal, the robotic UAV platforms are fitted with the necessary probing equipment: either a sample collector probe which picks water samples around $\sim 400 \mathrm{~cm} 3$ to bring them to a delivery station; or a multiparametric probe sensor to sample the available parameters online while in flight.

Thus, a human controller who operates the system is able to set a list of sampling tasks. Each one of these tasks is composed of the coordinates of a point in one of the basins, where the sampling has to take place, and a set of instructions or restrictions related to the sampling, including which parameters are desired to measure, what time or period should the measurements or samples to be taken, whether is required to keep the sample or a single measurement is enough, and any other features. The sampling system then checks the available UAV sampling platforms, receiving from them updates on their status (including values as battery charge available), and tries to plan a 
sampling solution which satisfies the list of sampling tasks. For a mission where there is no need to do laboratory work, i.e., the metrics to be analysed can be obtained with the multiparametric sensor probe and there is no requirement to keep or validate the sample at a later point, a UAV platform with the multiparametric probe will be sent, and the results from the analysis will be provided through a VPN over a 4G link even before the UAV has returned and landed (see Fig.2,left).



Fig. 2. Left) UAV network sampling system for sampling missions using multiparametric probe. Right) UAV network sampling system for sample capture mission

When there are conditions that makes the use of a multiparametric probe unsuitable (measurements that require long time to be measured, for instance hours or days for the consumed oxygen, or when the normative requires a contrast analysis with the same sample, etc...), a similar UAV with a sample collector probe (described in Water Sampling with a UAV section) will be sent. The operations of these UAVs are more complex, as the deployment of a probe that can be empty or filled with water, modifying the dynamic behaviour of the UAV. To fill it, the UAV submerges the probe into the open air basin by floating above the water, as the sample collection probe is rigidly solidary with the UAV. After enough time to fill the probe has passed, the UAV flies to a delivery station, where it releases the magnetic valve which sustains the probe, and also can land to be serviced (Fig. 2,right).

\section{System and Hardware Structure}

The system proposed is largely based in off-the-shelf (OTS) commercially available technologies for hardware, and open source software, reducing development and operational costs for the deployment of the prototype. This system is managed from a central control system, which is deployed in an ordinary PC, thus allowing to manage a network of sample collector UAVs and localization beacons. A single sample collector UAV prototype has been developed and built, based on a custom-design of UAV, with high power-to-weight ratio in order to be able to operate moderately heavy payloads affected by dynamics forces.

The sample collector UAV was designed as a quadcopter 12 at X4 configuration, due its stability and performance, with a length of $0.664 \mathrm{~m}$ between rotor axes. The propellers are standard 18" build in carbon fiber, with T-Motor MN4014 actuators. These rotors are controlled at low level by a set of 4 T-Motor AIR 40A electronic speed controllers (ESC), which are connected to a standard power supply board paired to a Pix- 
hawk 2.4.8 flight management unit (FMU). The power supply board is connected to a custom power system, feed by two $6 \mathrm{~S} 10,000 \mathrm{mAh}$ batteries. The custom power system allows several functions, like battery switch, balanced battery charge, and producing different voltages with a set of buck converter circuits.

The Pixhawk FMU is connected to a EGNOS SBAS13 enabled Ublox GPS, an internal IMU, a Lightware SFC10/B LRF set as altimeter, LRF two radio transceivers: a 915Mhz radio telemetry module; and a Futaba 2008RB 2.4Ghz to enable direct manual control during experimental validation of newly developed features. To run all the sensors and hardware the Pixhawk is set with the PX4 flight stack14, providing all the basic flight capabilities, included some autonomous flight capabilities, like a return-tohome (RTH) function, and take-off and landing using a Tarot landing set. This quadcopter setup has a maximum take-off weight of $13.9 \mathrm{~kg}$, with a payload weight ranging between 3.9 and $4.55 \mathrm{~kg}$ depending on the presence and configuration of a safety cage.



Fig 3. Hardware architecture of the prototype sample collector UAV developed. Not all the described components will be used concurrently or during standard operations.

This base UAV was enhanced with robotic capabilities after the addition of several sensors and an Odroid X4 single board computer (SBC). This SBC deploys a Exynos 5522 octacore based on ARM designs (comparable to a not so new high end smartphone in computational power), and presents several USB and serializable interfaces, including a GPIO pinout. This allows to connect several sensors and devices, including: a USB 3.0 camera (up to 5MP, usually set a VGA resolution to ease bandwidth usage during monitoring), a SBAS (EGNOS network) receiver, a network beacon transceiver, and a set of 4 MaxBotix ultrasonic sensors to detect near-collisions and obstacles. All this additional hardware allows fully autonomous flight operations in known prepared environments. The final addition to the UAV system is the sampling system, with two different options: deployment of a multiparametric probe or the de- 
ployment of a custom sample collector probe, described at a later section. A full schema of the computing architecture and all the devices connected can be seen in Fig. 3.

Given the complexity of the system described, it is useful to consider the distributed sampling network developed in terms of a set of different subsystems, with each subsystem comprised of both the hardware and software components responsible for a task. The hardware level components are generally organized according physical restrictions and specifications, while at software level, a multi-agent system (MAS) approach, based on the methodology described in [14], was considered the most convenient approach. Using a MAS design abstraction allows produce software level components with great modularity and robustness, which are integrated with the hardware level components through a set of low-level routines and drivers, which build an interface layer. In terms of software implementation, the multi-agent architecture uses a framework based on ROS middleware. Using a ROS framework allows quickly deploying new software, organized in executable units called "nodes", easing the issues of setting up and configuring communications between processes distributed in different machines. Thus, for each subsystem, one or more agents implemented in the ROS framework perform the high level functions and processes (including communications between them), and operate the hardware level components by communicating with the interface level layer.

Notice that several systems will be present in each instance of the hypothetical sampling network, i.e., for each of the UAV deployed; while other will work akin a singleton class, with only one instance for the whole network sampling system. System Design and Software Architecture

The different software components used in the proposed system can be described as a set of Agents which are organized according to a multi-agent system (MAS) architecture, which is largely implemented over a ROS framework9 (using ROS Kinetic Kame). This Multi-agent system is a convenient abstraction, providing a virtual infrastructure to organize and design the high level software capabilities. The ROS framework is run in an ODROID SBC deployed at the sample collector UAV, and the PC machine/s present at the main control subsystem. For the sake of compatibility all the hardware using ROS runs an Ubuntu 16 based distribution of Linux, hosting both the ROS framework, the software agents and all the required drivers and low level routines for communication and actuation. The fight management unit also runs several pieces of software at agent level, those closely related with the avionics systems of the UAV, and usually closely tied to the low level control routines communicating with the hardware level. These two devices communicate through a serial port in the FMU connected to the GPIO pinout in the ODRIOD. This architecture allows for perception and high level processes and agents to use the resources of the more powerful SBC, while the agents and resources needed to keep the sample collector UAV flying safely are isolated in the FMU, thus ensuring their performance and availability.

Note that a ROS framework usually requires that a single "ROS master node" (being a node the basic execution unit that communicates with other nodes through ROS) acts as a "master agent" managing all the routing and initial steps of every communication, and providing a unified clock for the system. This centralized architecture presents a critical fail point in this feature, as failure of this "node" usually invalidates the whole network. Moreover, although this can be managed in certain network environments, it 
becomes an unsolvable issue when the communications between the different physical elements of the system cannot be guaranteed, i.e.: deploying the master "node" in the ground or user stations at the main control system would endanger any sample collector UAV unit in flight if the 4G communication becomes unviable; while deploying it in a sample collector unit can risk other units (if they were present) and render the main control subsystem powerless.

In order to avoid the commented issues related to a master "node" acting as master agent, several master agents will be deployed using the multimaster FKIE tool. This allows to deploy multiple ROS master "nodes", and managing synchronization and coordination between them. So, from the MAS point of view, there will be a central master agent and a local master agent per UAV. The main difference between these central and local master agents resides in that the central master agent ensures coordination between the main control subsystem devices and every available UAV, while the local master agents only coordinate with the central master agent, and not between each other. This architecture ensures that the local ROS framework of an UAV will remain operative even if the connection with the ground station is not available, while isolating each UAV's communications from each other, to avoid sharing unnecessary ROS coordination data between them (which can require a noticeable bandwidth consumption, as discussed in [16] [17] [18]).

\section{$4 \quad$ Experimental Results}

\subsection{Water Sampling with UAV}

The hardware design and software architecture described in previous sections consider two different available setup options to sample the open air basins:1) using a commercial OTS multiparametric probe sensor; 2) deploying a custom designed sample collection device, able to hold over $400 \mathrm{~cm} 3$.

The first option presents two main advantages: it is an existing technology, widely supported, already integrated into PC environments, and from a flight and control point of view, a hanging solid body with fixed mass is a problem already solved by research in with several strategies. The second option also presents its own weaknesses and strengths: deployment of a hardware system able to collect water samples from an UAV robotic platform is cheap under the right designs, and although it is not able to sample on the fly, it can be used in processes where the sample must be kept for periods of time, or requires any kind of processing before performing analytics tests. Note that the utilization of the sample collector probe implies that the mass of the UAV platform, and its distribution (so inertia) will vary dynamically during the collection and return flight, introducing disturbances in the low level control loops.

The proposed sample collection probe was developed and built in our laboratory. The probe is able to hold $\sim 0.41$ according to the fill tests, with a buoyancy valve that will lock the containment part as it floats to its top when filled. The probe is attached to the UAV by a ferromagnetic plate at its top, which is locked into a magnetic gripper. This magnetic gripper works with negative logic for safety, so in case of failure, the probe will be locked and cannot be released. The probe has to be submerged for $3 / 4$ of 
its height, so that waters reach the opposing entry holes, whose position ensure that the flow velocities of entering water induce negligible torque. To empty the probe, a screwcap at the bottom is used, which also allow retrieving the buoyancy valve for cleaning and maintenance.

\subsection{UAV Flight Validation System Identification}

A critical step in building any UAV platform is the setting and configuration of the avionics controller parameter, which have to be tuned individually for each design. From a robotics point of view, accurate modelling of the dynamic behaviour of the base UAV platform is critical to develop a robotics platform, as it is required for accurate localization and navigation operations (including trajectory planning). In order to satisfy this requirements, several indoor flight test were performed, in a motion capture testbed using an Optitrack ${ }^{\circledR}$ system (see Fig. 4). This system allowed to accurately validate the performance of the system according to the avionics controller parameters, and capture enough data to produce an accurate enough dynamic model to use in the robotics agents (localization and navigation) through parameter identification.

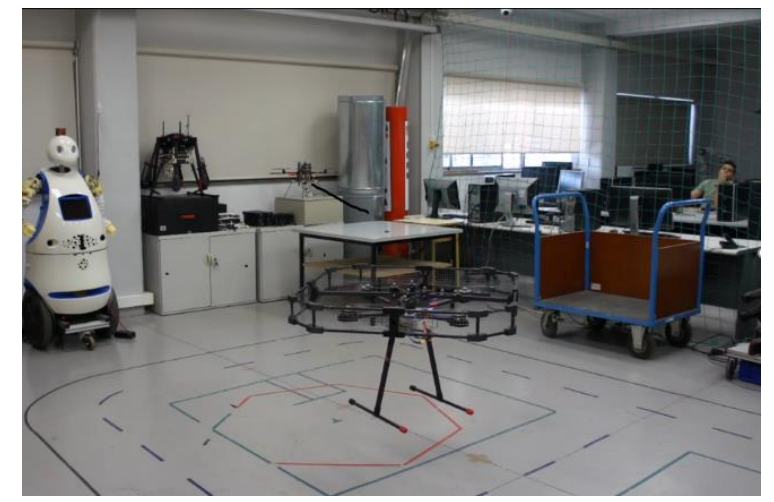

Fig 4. UAV prototype platform flying indoors in an Optitrack ${ }^{\circledR}$ testbed. The UAV is equipped with a carbon fiber cage with a light metal grid for safety reasons, as the system is still in development stages.

\subsection{UAV Localization}

For any autonomous UAV system one of the most complex issues is always the localization problem. Commercial solutions with high accuracy are available, but present several challenges due the trade-offs between weight, accuracy, performance and economical costs. Though differential GPS can produce the most accurate results, its use requires deployment of expensive equipment both in the UAV and as a part of infrastructure, and the rate of its data $(5 \sim 10 \mathrm{~Hz})$ is not enough to perform robust localization and navigation for UAVs. All the other GPS-based solutions, like SBAS augmentation with the EGNOS network available also share this specific issue. Notice that for the proposed system, especially for the scenario considering the utilization of the sample 
collector probe, a correct estimation of height is critical. As it can be observed after the experiments, neither of the GPS-based approaches produce an accurate enough height estimation to perform the required manoeuvres to sample the basins flying close to the surface. To solve this issue, an LRF (laser range finder) altimeter was deployed. The avionics implements a low level extended Kalman filter [17] [18] to integrate results of the EGNOS-enabled GPS with measurements from the internal IMU and the laser altimeter.

Though the results produced by the avionics system fusing readings from all the sensors, the availability of the EGNOS enhancement is not guaranteed, as it has been widely discussed in literature [19]. Moreover, while the avionics system can reduce the height error thanks to the altimeter, the accuracy in the planar positioning is not good enough for the landing and sample collector probe delivery tasks. To solve this, the additional localization tasks are performed at the localization agent level. Using data from the camera agent, a fiducial marker detection process is used to achieve centimetre level accuracy in marked areas, allowing proper landing and probe delivery operations. To deal with the eventuality of EGNOS shortage, a system of beacons based in the TI chip CC2530 has been tested [15]. This component implements a full SoC solution (system on a chip) for ZigBee-based applications, and its low cost and low-power consumption makes it ideal for developing inexpensive, easy to deploy and maintain beacon networks. This system would be deployed around the open air basin, to improve UAVs localization during EGNOS shortages. To perform the test, a set of 6 beacons was deployed in a planar configuration covering an area of $30 \mathrm{~m}$ per $15 \mathrm{~m}$. These tests reported errors within a compatible range of those reported within previous literature [19] [20] [21] when accounting for differences in the scenarios. Still, active research in the field is producing new solutions [22] that could improve the results.

\section{Conclusions}

This works proposes a solution to the problem of automatizing the sampling and monitoring of a wastewater treatment plant. The solution proposed is the utilization of a network of sampling UAVs with different sampling methods and capabilities. This UAV sampling network would act as a virtual sensor network composed of hundreds of multiparametric sensors/sample capturing probes, as it is capable of operating at any point of an accumulated area over $11,200 \mathrm{~m} 2$. The sensing UAV network system proposed is designed as a multi-agent system, built over a ROS framework, which provides several advantages derived from the fact that each agent is responsible of a task and has its own status and agency, producing increased robustness, modularity, improved upgradability, and ease of maintenance.

With respect to the solution proposed as a system, several advantageous contributions over author's previous works have been presented. First of all, the proposed system is much better from a design and implementation point of view, with better isolation between tasks. The system architecture is fully scalable, so new sampling UAV can be added to the sampling network system with ease, as the MAS design allows to accommodate them with minimal changes in the implementation. Replicating the UAV sample collector is a trivial task, and migrating the MAS system into an upgraded 
hardware platform should not be complex, with the only challenges present in the mechanical integration of sampling devices.

In terms of the sampling process, the designed probe has been tested in simulated tanks, and its performance meets the specifications. The design with two entry points allows to reduce possible torques to negligible values, and allows the correct filling of the sample container. The buoyancy valve presents no losses under normal operation, and under shock circumstances, losing a sample is irrelevant, given other risks incurred during an UAV crash.

The actual performance of the robotic system has been only tested in terms of localization. Being one of the hardest challenges for $\mathrm{UAV}$, achieving a satisfactory solution in terms of accuracy and performance without expensive equipments (be it in economical or payload terms), is always complex. Several solutions have been tested, with validation data provided by a highly accurate DGPS processed off-line. Tests revealed that SBAS-enhanced GPS with the EGNOS network can provide accurate enough data, but is not reliable enough to automate a system, so an alternative beacon method was tested. The beacons present slightly less accuracy, but results are much more robust, and are not subject to unpredictable disturbances. The biggest issue that remains to be addressed in the localization problem is replacing the laser range finder LRF altimeter by a device able to operate properly over the water, and reduce its impact.

Future works must address some specific challenges still pending, starting with a robust flight controller able to maintain the UAV over water while the sample collector fills the bottle with water to be analysed. The risks implied by using a multiparametric sensor probe can be easily managed at the hardware integration step, as the sensor can hang as much as needed, acting as an inverted pendulum (which has been solved for years). This specific challenge presents a strong dependency with the localization problem, as the controller must account for which specifications are guaranteed by the localization agent in terms of accuracy, rate and robustness. Finally, the sampling scheduler part of the sampling network manager agent can be further developed, as of now its implementation assumes that the UAVs are uniform except for the specific sampling device, and its performance is also uniform (e.g., it could not account that batteries and other components degrade with time dealing different performances and capabilities).

Acknowledgments. This research has been funded by AEROARMS EU Project H2020-ICT-2014-1-644271.

\section{References}

1. Heyer C. Human-robot interaction and future industrial robotics applications. In: 2010 IEEE/RSJ International Conference on Intelligent Robots and Systems (IROS). 2010, pp. 4749-4754.

2. Ping JTK, Ling AE, Quan TJ, et al. Generic unmanned aerial vehicle (UAV) for civilian application-A feasibility assessment and market survey on civilian application for aerial imaging. In: 2012 IEEE Conference on Sustainable Utilization and Development in Engineering and Technology (STUDENT). 2012, pp. 289-294.

3. Loh R, Bian Y, Roe T. UAVs in civil airspace: Safety requirements. IEEE Aerospace and Electronic Systems Magazine 2009; 24: 5-17. 
4. Freeman P, Balas GJ. Actuation failure modes and effects analysis for a small UAV. In: 2014 American Control Conference. 2014, pp. 1292-1297.

5. Niazi M, Hussain A. Agent-based computing from multi-agent systems to agent-based models: a visual survey. Scientometrics 2011; 89: 479.

6. Iñigo-Blasco P, Diaz-del-Rio F, Romero-Ternero MC, et al. Robotics software frameworks for multi-agent robotic systems development. Robotics and Autonomous Systems 2012; 60: 803-821.

7. Conte G, Scaradozzi D, Mannocchi D, et al. Development and Experimental Tests of a ROS Multi-agent Structure for Autonomous Surface Vehicles. Journal of Intelligent \& Robotic Systems 2018; 92: 705-718.

8. Langley P, Laird JE, Rogers S. Cognitive architectures: Research issues and challenges. Cognitive Systems Research 2009; 10: 141-160.

9. Quigley M, Conley K, Gerkey B, et al. ROS: an open-source Robot Operating System. In: ICRA workshop on open source software. 2009, p. 5.

10. Guerra E, Bolea Y, Grau A, et al. A solution for robotized sampling in wastewater plants. In: IECON 2016 - 42nd Annual Conference of the IEEE Industrial Electronics Society. 2016, pp. $6853-6858$.

11. Urban Waste Water Directive - Environment - European Commission, $\mathrm{http} / / /$ ec.europa.eu/environment/water/water-urbanwaste/legislation/directive en.htm (accessed 28 February 2019).

12. Mahony R, Kumar V, Corke P. Multirotor Aerial Vehicles: Modeling, Estimation, and Control of Quadrotor. IEEE Robotics Automation Magazine 2012; 19: 20-32.

13. Subirana JS, Zornoza JMJ, Hernández-Pajares M. $\rightarrow$ GNSS DATA PROCESSING. 238.

14. Meier L, Honegger D, Pollefeys M. PX4: A node-based multithreaded open source robotics framework for deeply embedded platforms. In: 2015 IEEE International Conference on Robotics and Automation (ICRA). 2015, pp. 6235-6240.

15. CC2530 Second Generation System-on-Chip Solution for 2.4 GHz IEEE 802.15.4 / RF4CE / ZigBee | TI.com, http://www.ti.com/product/CC2530 (accessed 28 February 2019).

16. Hernandez S, Herrero F. Multi-master ROS systems. IRI-TR-15-1, http://hdl.handle.net/2117/80829 (2015).

17. Jun Zeng, Minbo Li, Yuanfeng Cai. A Tracking System Supporting Large-Scale Users Based on GPS and G-Sensor, 2015, https://journals.sagepub.com/doi/abs/10.1155/2015/862184 (accessed 28 February 2019).

18. Trujillo J-C, Munguia R, Guerra E, et al. Cooperative Monocular-Based SLAM for MultiUAV Systems in GPS-Denied Environments. Sensors 2018; 18: 1351.

19. Liu J, Chen R, Chen Y, et al. Performance Evaluation of EGNOS in Challenging Environments. Journal of Global Positioning Systems 2012; 11: 145-155.

20. Adnan T, Datta S, MacLean S. Efficient and accurate sensor network localization. Personal and Ubiquitous Computing 2014; 18: 821-833.

21. Guo Y, Liu X. A Research on the Localization Technology of Wireless Sensor Networks Employing TI's CC2530 Instrument. IEEE, pp. 446-449.

22. Thammavong L, Khongsomboon K, Tiengthong T, et al. Zigbee wireless sensor network localization evaluation schemewith weighted centroid method. MATEC Web Conf 2018; 192: 02070. 